\title{
EXPERT REVIEWS \\ The role of targeted therapy for gastrointestinal tumors
}

Expert Rev. Gastroenterol. Hepatol. 8(8), 875-885 (2014)

\section{Christian Rolfo* ${ }^{1}$ Giuseppe Bronte ${ }^{2}$ Giovanni Sortino2, Konstantinos Papadimitriou", Francesco Passiglia ${ }^{1,2}$, Eugenio Fiorentino ${ }^{2}$, Ghada Marogy ${ }^{1}$, Antonio Russo ${ }^{2}$ and Marc Peeters ${ }^{1}$}

${ }^{1}$ Oncology Department, University Hospital Antwerp UZA, University of Antwerp, Wilijkstraat 10, 2650, Antwerp, Belgium

${ }^{2}$ Department of Surgical, Oncological and Oral Sciences, University of

Palermo, Via del Vespro 129, 90127 Palermo, Italy

*Authors for correspondence: Tel.: +32 38213646 christian.rolfo@uza.be; antonio.russo@usa.net

Many targeted drugs have been studied to target the molecular pathways involved in the development of gastrointestinal cancers. Anti-VEGF, anti-EGFR agents, and recently also multi-kinase inhibitor regorafenib, have already been available for the treatment of metastatic colorectal cancer patients. To date, Her-2 positive, gastric cancer patients, are also treated with trastuzumab, while the multi-targeted inhibitor, sorafenib, represents the standard treatment for hepatocellular carcinoma patients. Finally, sunitinib and everolimus, have been approved for the treatment of the neuroendocrine gastroenteropancreatic tumors. Actually a great number of further drugs are under preclinical and clinical development. The aim of this review is to provide a comprehensive overview of the state of art, focusing on the new emerging strategies in the personalized treatment of gastrointestinal tumors.

\section{KeYWORDS: colorectal cancer $\bullet$ gastric cancer $\bullet$ gastrointestinal tumors $\bullet$ hepatocellular carcinoma}

- neuroendocrine tumors $\bullet$ targeted therapy

\section{The main pathways involved in gastrointestinal tumors VEGF/VEGFR pathway}

Tumor-related angiogenesis has a predominant role in gastrointestinal (GI) cancers. This process involves the formation of new blood vessels within the tumor to provide nutrients and oxygen. VEGF is an endothelial cell-specific mitogen that induces angiogenesis and is expressed in various human tumors [1].

VEGF was first isolated in 1983 as a factor leading to increased vascular permeability in tumors. VEGF-A is the most important member of a family of homodimeric glycoproteins. This family of proteins is structurally related to the PDGF; and it also includes PIGF, VEGF-B, VEGF-C, VEGF-D and VEGF-E. These growth factors bind selectively, but with different affinity, to at least five distinct receptors. In particular, VEGF-A binds to receptors VEGFR-1 and VEGFR-2. Preclinical studies in mice showed that the inactivation of VEGFR-1 and VEGFR-2 as well as neuropilin-1 induces impairment of blood vessel formation and embryonic lethality, supporting the important role of VEGF-A for a proper vascular development [2-4].

Both normal function, including embryogenesis, the female reproductive cycle, pregnancy, wound healing and disease-related phenomena, such as tumor growth, diabetic retinopathy and ischemic diseases, are subsequent to the upregulation of the VEGF/VEGFR system [5]. In the search for the mechanisms and factors able to regulate VEGF gene expression during these processes, many cytokines and growth factors are involved [6]. For example, TNF- $\alpha$ and bFGF are able to induce VEGF gene expression in vitro, but also glucose deficiency has this effect. However, the main factor able to regulate its expression is hypoxia [7-9]. As a consequence of hypoxia development, specific hypoxiainducible transcription factors, HIF-1 and HIF-2, are activated and induce VEGF expression. In addition, VEGF upregulation during hypoxia is also achieved by an increase in the stability of its mRNA51 and by the efficient hypoxic translation of the VEGF mRNA, which is mediated by an internal ribosomal entry site [10-12].

In endothelial cells, VEGFR-2 is considered to be the major signaling receptor, while VEGFR-1 acts as a sink to trap an excess of VEGF. Endothelial proliferation is mediated via the Ras-Raf-MAP kinase pathway, while protein kinase $\mathrm{C}$ activation is involved in endothelial migration and vascular permeability. The role of VEGF as a survival factor for endothelial cells is mediated by the PI3K-AKT signaling pathway [13]. 


\section{Table 1. Approved targeted agents in advanced gastrointestinal tumors.}

\begin{tabular}{|c|c|c|c|c|c|}
\hline Type of tumor & Targets & Drugs & Setting & Overall survival & Ref. \\
\hline \multirow[t]{5}{*}{ Colorectal cancer } & \multirow[t]{2}{*}{ VEGF } & Bevacizumab & $\begin{array}{l}\text { First-line, } \\
\text { Second-line } \\
\text { Beyond PD }\end{array}$ & $\begin{array}{l}21.3 \text { months } \\
12.9 \text { months } \\
11.2 \text { months }\end{array}$ & {$[21,29,31]$} \\
\hline & & Aflibercept & Second-line & 13.5 months & [32] \\
\hline & \multirow[t]{2}{*}{ EGFR } & Cetuximab & First-line (KRAS-wild-type) & 23.5 months & [23] \\
\hline & & Panitumumab & $\begin{array}{l}\text { First-line (RAS-wild-type), } \\
\text { second-line (KRAS-wild-type) }\end{array}$ & $\begin{array}{l}26.0 \text { months } \\
14.5 \text { months }\end{array}$ & {$[24,33]$} \\
\hline & $\begin{array}{l}\text { VEGFR, BRAF, } \\
\text { KIT, RET, PDGFR }\end{array}$ & Regorafenib & Third-line & 6.4 months & [34] \\
\hline Gastric cancer & HER2 & Herceptin & First-line & 13.8 months & [38] \\
\hline Hepatocellular carcinoma & $\begin{array}{l}\text { VEGFR, BRAF, } \\
\text { KIT, RET, PDGFR }\end{array}$ & Sorafenib & First-line & $\begin{array}{l}10.7 \text { months } \\
6.5 \text { months }\end{array}$ & {$[39,40]$} \\
\hline \multirow{2}{*}{$\begin{array}{l}\text { Neuroendocrine } \\
\text { gastroenteropancreatic } \\
\text { tumors }\end{array}$} & $\begin{array}{l}\text { VEGFR, BRAF, } \\
\text { KIT, RET, PDGFR }\end{array}$ & Sunitinib & Second-line & N.A & [45] \\
\hline & mTOR & Everolimus & Second-line & N.A & [46] \\
\hline
\end{tabular}

\section{EGFR/HER2 pathway}

The family of ErbB receptors includes four homologous receptors: the EGFR (ErbB1/EGFR/HER1), ErbB2 (HER2/ neu), ErbB3 (HER3) and ErbB4 (HER4) [14]. The EGFR is a membrane-bound tyrosine kinase that contributes to signaling cascades with multiple procarcinogenic effects including cell proliferation, motility, adhesion, invasion, cell survival and angiogenesis.

EGFR overexpression has been detected in several human cancers, including colorectal cancer, and it is related to progression and metastasis. HER2 overexpression or gene amplification has been observed in multiple malignancies, including gastric cancer. These receptors are composed of an extracellular binding domain, a transmembrane lipophilic segment and an intracellular protein TK domain. A cross-talk between the various ErbB receptors has been already known. It regulates the cellular effects mediated by these receptors [15].

At least six different ligands, known as EGF-like ligands, can bind to EGFR. These ligands include EGF, TGF- $\alpha$, amphiregulin, heparin-binding EGF, betacellulin and epiregulin. A second class of ligands, collectively termed heregulins, binds directly to HER3 and/or HER4.

After their binding to ErbB receptors these are activated by the dimerization between two identical receptors (homodimerization) or between different receptors of the same family (heterodimerization). This dimerization allows tyrosine autophosphorylation and subsequently the recruitment and phosphorylation of several intracellular proteins, which mainly belong to the Ras-Raf-MAPK and the PI3K-Akt pathways. RAS protein function is normally regulated by cycling between inactive GDP-bound and active GTP-bound forms. Signaling is terminated when RAS-GTP is hydrolyzed to the RAS-GDP inactive complex by GTPaseactivating proteins. Activated RAS recruits Raf protein to the cell membrane and phosphorylates it, triggering serine-threonine kinase activity of various proteins [16]. Finally, active MAPKs can translocate to the nucleus, where they regulate the activity of several transcription factors for the expression of multiple genes of survival and proliferation [17].

\section{PTEN-PI3K-AKT-mTOR pathway}

PI3Ks are a family of lipid kinases that phosphorylate the 3 '-hydroxyl group of phosphoinositides with conversion of phosphatidylinositol-4,5-biphosphate (PIP2) to phosphatidylinositol3,4,5-trisphosphate (PIP3). PIP3 is a critical second messenger that activates AKT (also known as protein kinase B) through phosphorylation. Once activated, phospho-AKT phosphorylates up to 100 other proteins, including the mTOR. mTOR complexes with regulatory associated protein of mTOR and rapamycin-insensitive companion of mTOR to form mTOR complex 1 (mTORC1) and mTORC2, respectively [18,19].

\section{US FDA-approved targeted drugs}

Nowadays, targeted drugs have been developed with the perspective of sparing cancer patients from chemotherapy-related toxicity. However, some of these agents showed their best efficacy in combination with chemotherapy regimens. A lot of new targeted drugs are still under development, but monoclonal antibodies and small molecule inhibitors are actually available after the US FDA approval to treat GI cancers (TABiE 1). These agents are able to target VEGF/VEGFR or EGFR/HER2 pathways.

\section{Colorectal cancer}

Recently, the combination of targeted drugs and irinotecan/ oxaliplatin-based chemotherapy regimens has improved the efficacy of standard treatment for colorectal cancer patients. Bevacizumab is a humanized monoclonal antibody (mAb), 
which inhibits angiogenesis by its binding to VEGF. Various Phase III trials evaluated patients with previously untreated metastatic colorectal cancer (mCRC) who were randomly assigned to receive irinotecan, bolus fluorouracil and leucovorin (IFL) plus bevacizumab or IFL plus placebo. In this pivotal trial, the median duration of survival was 20.3 months in the group receiving IFL plus bevacizumab versus 15.6 months in the control group. These results were quite impressive [20]. Other trials have failed to demonstrate the same statistically significant results in survival, particularly with other backbone regimens, such as isolated capecitabine or oxaliplatin-containing regimens like FOLFOX or XELOX plus placebo or bevacizumab in first-line mCRC (median overall survival [mOS] 19.9 vs 21.3 months in bevacizumab group) [21]. Cetuximab and panitumumab are anti-EGFR mAbs delivered alone or in combination with chemotherapy. To date, the main factor conditioning their use is KRAS and NRAS mutation status. Even BRAF mutation seems to have a role as a predictive factor for the efficacy to anti-EGFR mAbs, but its prognostic role appears predominant. After the detection of somatic RAS gene mutations, each oncologist is able to know if a mCRC patient could have a benefit from the combination of the anti-EGFR mAbs with standard chemotherapy doublets [22]. In fact for RAS wild-type patients, the benefit in OS was observed for both these two combinations: FOLFIRI + cetuximab, as shown in the CRYSTAL trial [23] and FOLFOX + panitumumab, as reported in the PRIME trial after the specific evaluation of both KRAS and NRAS gene mutations [24]. Some concerns have been reported in guidelines for the combination of oxaliplatin-based regimens with cetuximab. It showed no additional benefit in OS in the OPUS trial and in some studies more toxicity has been recorded, as reported in NORDIC VII and COIN trials [25-27]. For this reason, in KRAS or NRAS mutated mCRC patients, who cannot receive anti-EGFR mAbs, FOLFOX + bevacizumab represents the best option, instead of FOLFOX or FOLFIRI alone, because it can delay progression even though it cannot reduce the risk of death [28]. Nowadays, targeted therapy has also a role for the second-line treatment. It is mainly influenced by the scant evidences about a re-challenge with the same chemotherapeutic regimen. The re-challenge with bevacizumab has been shown to give further benefit [29]. For the re-challenge with cetuximab, some intriguing results have been reported till now, but strong evidences are still not available [30]. For this reason, the more valid options for second-line treatment are represented by FOLFOX + bevacizumab for those RAS wild-type patients who received FOLFIRI + cetuximab as first-line treatment, and FOLFIRI + aflibercept after first-line treatment with FOLFOX + panitumumab. The first second-line option is supported by the results from E3200 trial, which obtained survival benefit through the addition of bevacizumab to oxaliplatinbased treatment in those patients who did not receive bevacizumab before [31]. The other one is based on the results of VELOUR trial, which reported an OS benefit when aflibercept was added to FOLFIRI after a previous treatment with an oxaliplatin-based regimen [32]. Aflibercept is a recombinant fusion protein consisting of VEGF-binding portions from the extracellular domains of VEGFR-1 and VEGFR-2, which are fused to the Fc portion of the human IgG1 immunoglobulin. In RAS mutated mCRC patients who received an oxaliplatinbased regimen plus bevacizumab as first-line treatment, two options are available on the basis of evidence-based findings from Phase III trials with OS benefit. These chances include both FOLFIRI + aflibercept according to the VELOUR trial's results and FOLFIRI + bevacizumab as highlighted by the ML18147 trial. Both these two combination regimens are superior to FOLFIRI alone in terms of OS. Moreover, panitumumab-FOLFIRI represents a valid option for wildtype KRAS, mCRC patients, who received an oxaliplatinbased regimen plus bevacizumab as first-line treatment [33]. A further effective option with a targeted drug could be offered to those patients, who have an acceptable general health status after the first- and second-line treatments. Indeed, regorafenib, a BRAF inhibitor, showed an OS benefit over placebo in the Phase III CORRECT trial for unselected patients who previously were treated with irinotecan- and oxaliplatin-based regimens [34]. Recently, FIRE-3 trial, which compares the combination of chemotherapy and bevacizumab with the same chemotherapy and cetuximab in KRAS wildtype mCRC patients, provided interesting results useful for the decision-making in first-line treatment. Indeed, cetuximab achieved a better OS compared with bevacizumab without difference in progression-free survival (PFS). These findings are controversial and should be better clarified with further studies, since PFS and OS were not the primary end points in this trial [35].

\section{Gastric cancer}

Advanced gastric cancer (aGC) patients obtain an improvement in survival from combination chemotherapy more than from mono-chemotherapy [36]. About 20\% of gastric and esophagogastric junction tumors overexpress HER2. This finding provided a rationale to investigate a biological target therapy as trastuzumab, a mAb directed against the extracellular domain of HER2 protein, also in gastric cancer [37]. ToGA trial was designed to explore the efficacy of trastuzumab. It is an open-label Phase III trial, which investigated the survival benefit induced by trastuzumab in combination with chemotherapy for first-line treatment of HER2-positive advanced gastric or gastroesophageal junction (GEJ) cancer. Median OS was favorable for trastuzumab plus chemotherapy arm (13.8 vs 11.1 months), with $26 \%$ reduction in the death rate. Anyway, the benefit of trastuzumab has been shown only for those patients whose tumors were at immunohistochemistry (IHC) 3+ or IHC 2+ and positive at fluorescence in situ hybridization, but no survival benefit was found in IHC 0 or $1+$ and positive at FISH [38]. These results prompted the admission of this combination as a new standard therapy for patients affected by inoperable/metastatic gastric or GEJ cancer. 


\section{Hepatocellular carcinoma}

Sorafenib is a multikinase inhibitor that blocks tyrosine kinase activity in membrane receptors, such as VEGFR and PDGFR, and determines the inhibition of tumor vascularization and proliferation. This drug was approved for systemic treatment of patients with advanced inoperable hepatocellular carcinoma (HCC), Child-Pugh class C patients. SHARP [39] and Asia-Pacific trials [40] showed a statistically significant 3 and 2 months improvement of mOS, respectively by sorafenib compared with placebo (SHARP: 10.7 vs 7.9 months, hazard ratio [HR]: 0.69; 95\% CI: $0.55-0.87 ; \mathrm{p}<0.001$; Asia-Pacific: 6.5 vs 4.2 months, HR: 0.68 ; $95 \%$ CI: 0.50 $0.93 ; \mathrm{p}=0.014)$. In both these studies, a benefit from sorafenib was also observed for median time to progression (TTP) (SHARP: 5.5 vs 2.8 months, HR: 0.58 ; 95\% CI: $0.45-0.74$; p < 0.001; Asia-Pacific: 2.8 vs 1.4 months, HR: 0.57 ; $95 \%$ CI: $0.42-0.79 ; \mathrm{p}=0.0005)$. Therefore, sorafenib provides a good efficacy in terms of TTP and OS with an acceptable tolerability profile. These results could be translated into an increase of about $11 \%$ for 1-year survival rate and about $31 \%$ of death risk reduction. This benefit is also maintained if these data are adjusted for some prognostic factors by multivariate analysis, including electrocorticography performance status, vascular invasion, extrahepatic extension, Child-Pugh status, $\alpha$-fetoprotein, serum albumin, alkaline phosphatase and bilirubin. However, an improvement of response rates was not observed in these trials. In fact, partial responses were just 7 out of $299(2 \%)$ in the SHARP trial and just 5 out of 150 patients (3.3\%) in the Asia-Pacific trial. No complete responses were observed. Recently, sorafenib has also been proposed for use with caution in Child-Pugh class $\mathrm{B}$ patients, in particular if bilirubin levels are elevated [41]. It is approved for metastatic, unresectable and extensive HCC without suitability for liver transplantation. Those patients with local disease could also be treated with sorafenib, if they are not operable because of worse performance status or comorbidity.

\section{Neuroendocrine gastroenteropancreatic tumors}

Some years ago, the treatment options for neuroendocrine gastroenteropancreatic tumors were quite limited. Recently, two targeted agents, sunitinib and everolimus, achieved antitumor activity and improvement of PFS. For these reasons, these two drugs have been approved for the systemic treatment of these malignancies.

Sunitinib is an oral small-molecule, multi-targeted receptor tyrosine kinase inhibitor (TKI) for VEGFR and PDGFR. It plays a role in both tumor angiogenesis and tumor cell proliferation. The tumor shrinkage induced by the concomitant inhibition of these two target proteins is mediated by both reduced tumor vascularization and cancer cell death. Other target proteins have been identified, which include KIT, RET, CSF-1R and flt3 [42-44]. Sunitinib was studied in comparison with placebo in a multicenter randomized trial for pretreated patients with advanced pancreatic neuroendocrine tumors. This trial showed a PFS of 11.4 months for sunitinib versus 5.5 months in the control arm $(p<0.001)$. The difference in OS was not significant because a large proportion of patients progressing on placebo received sunitinib as subsequent treatment. The most frequent side effects of sunitinib include fatigue, diarrhea, nausea, anorexia, hypertension, a yellow skin discoloration, handfoot skin reaction and stomatitis [45]. Everolimus is a derivative of sirolimus, which inhibits the mTOR. As the other mTOR inhibitors, it acts on the mTORC1 protein and not on the mTORC2 protein. As a consequence, the kinase AKT is hyperactivated via an inhibition on the mTORC1-negative feedback loop, while not inhibiting the mTORC2-positive feedback loop. As TKIs it is orally delivered. It was studied in a multicenter trial (RADIANT-3) in comparison with placebo. The PFS was 11.0 months for everolimus versus 4.6 months for placebo $(\mathrm{p}<0.001)$. Furthermore, this outcome was confirmed as independent from prior chemotherapy or somatostatin ana$\log$ in a subgroup analysis. The most frequent adverse effects from everolimus were stomatitis, hyperglycemia and pneumonitis $[46-48]$.

\section{The development of new targeted drugs}

As reported here in GI tumors many targeted drugs are approved and available in clinical practice as monotherapy or combination with standard chemotherapy regimens. These agents target various components of VEGF/VEGFR and EGFR/HER2 pathways. Many studies are ongoing to improve the efficacy of treatment targeting these pathways and their downstream effectors. In the meanwhile, other signaling pathways are explored to find a role in carcinogenesis and cancer progression and potentially to discover new antitumor agents (Table 2).

\section{The improvement of previous treatments}

Both bevacizumab and cetuximab/panitumumab were tested in combination with other targeted agents for the treatment of refractory $\mathrm{mCRC}$ in Phase II trials. Bevacizumab plus everolimus combination was shown to be well tolerated in patients previously treated with bevacizumab in a single-arm Phase II trial. No complete or partial responses were seen. The median PFS interval was 2.3 months, $26 \%$ of patients achieved prolonged SD for $\geq 6$ months and $6 \%$ were on study for $>1$ year. However, some concerns are related to mucosal damage and/or wound healing. Bevacizumab plus everolimus appears to have modest activity in refractory $\mathrm{mCRC}$ in patients [49]. Since preclinical studies [50] showed a synergistic activity of cetuximab and erlotinib on growth inhibition in colon cancer cell lines, a Phase II trial was performed to test this combination. Response rate (RR) was $41 \%$ (95\% CI: 26-57\%) in KRAS-wild-type tumors, with a median PFS of 5.6 months (95\% CI: 2.9-5.6 months). There was no response in 11 patients with KRAS mutations. Frequent grade 3 and 4 toxicities were rash (48\%), hypomagnesemia (18\%) and fatigue (10\%) [51]. Some of these targeted agents were investigated in combination with chemotherapy. In 
particular, sunitinib was delivered in combination with FOLFIRI to mCRC Japanese patients in a single-arm Phase II trial. Median PFS did not meet the planned 35\% improvement compared with FOLFIRI alone. It was associated with a high incidence of grade $3 / 4$ hematologic toxicity impacting on dose intensity. Subsequently, a non-Japanese Phase III trial showed that sunitinib plus FOLFIRI is not superior to FOLFIRI alone with a worse toxicity profile [52]. Similarly, sorafenib was combined with FOLFOX6 in a randomized Phase IIb trial in comparison with FOLFOX6 alone for firstline treatment in mCRC. This study did not find a PFS benefit with the combination therapy. KRAS and BRAF status did not change this result [53].

In refractory mCRC patients, tivozanib, an oral VEGFR-1, $-2,-3$ inhibitor, was studied in combination with everolimus in a Phase Ib/II trial. This combination was well tolerated, achieving stable disease in $50 \%$ of patients. The most common grade $3 / 4$ adverse events were thrombocytopenia and hypophosphatemia [54].

Novel anti-angiogenic TKIs were compared with sorafenib for first-line treatment of advanced HCC in several Phase III trials. These include sunitinib and linifanib, which target VEGFR and PDGFR, and brivanib, targeting also FGFR. None of these novel anti-angiogenic TKIs has shown superior efficacy to sorafenib [55-57].

Brivanib was also compared with placebo for HCC after sorafenib failure in the BRISK-PS Phase III trial. It showed a higher overall response rate (ORR) (11.5 vs $1.9 \%)$ and a longer median TTP (4.3 vs 2.7 months; $\mathrm{p}=0.0001$ ), but did not significantly improve OS (9.4 vs 8.2 months; $\mathrm{p}=0.33$ ) [58].

Anti-angiogenic agents were also combined with erlotinib for HCC in the SEARCH Phase III trial. Sorafenib plus erlotinib were compared with sorafenib plus placebo [59]. No improvement in TTP and OS was found. Furthermore, in a Phase II trial, temsirolimus, a mTOR inhibitor, plus bevacizumab achieved an ORR of $30.7 \%$ with fair tolerability for patients in whom sorafenib failed [60].

The EGFR pathway has also been investigated for gastric and esophageal cancers. The SWOG 0127 study, a Phase II trial, showed no responses in patients with unresectable or aGC treated with erlotinib while those with GEJ cancers obtained a RR of 9\%. OS was 6.7 months for GEJ cancer patients versus 3.5 months for gastric once and also TTP was better for GEJ cancers (3.0 vs 1.7 months) [61]. Erlotinib with modifiedFOLFOX6 chemotherapy has been studied for patients with metastatic or advanced adenocarcinoma of the esophagus and GEJ obtaining a RR of $55 \%$, a median PFS of 5.5 months and a mOS of 11.0 months [62]. The combination of another TKI, gefitinib, with cisplatin and 5-fluorouracil chemotherapy plus radiotherapy, led to an improvement of OS in the neoadjuvant setting, even though not significant $(p=0.06)$ [63]. According to these data, we can assume that the different responses to TKI of GEJ carcinomas compared with stomach cancers may be related not only to anatomical differences or risk factors, but also to the activation of different molecular
Table 2. New targeted agents in clinical development in advanced gastrointestinal tumors.

\begin{tabular}{|c|c|c|c|}
\hline Type of tumor & Targets & Drugs & Ref. \\
\hline \multirow[t]{5}{*}{ Colorectal cancer } & mTOR & Everolimus & [49] \\
\hline & EGFR & Erlotinib & [51] \\
\hline & \multirow{2}{*}{$\begin{array}{l}\text { VEGFR, BRAF, } \\
\text { KIT, RET, PDGFR }\end{array}$} & Sunitinib & [52] \\
\hline & & Sorafenib & [53] \\
\hline & VEGFR & Tivozanib & [54] \\
\hline \multirow[t]{10}{*}{ Gastric cancer } & HER2 & Lapatinib & {$[66,67]$} \\
\hline & \multirow[t]{4}{*}{ EGFR } & Erlotinib & {$[61,62]$} \\
\hline & & Gefitinib & [63] \\
\hline & & Cetuximab & [68-71] \\
\hline & & Panitumumab & [72] \\
\hline & \multirow[t]{2}{*}{ VEGF } & Bevacizumab & [73-75] \\
\hline & & Ramucirumab & {$[78,79]$} \\
\hline & \multirow[t]{3}{*}{ c-MET } & Foretinib & [86] \\
\hline & & Rilotumumab & {$[87,93]$} \\
\hline & & Onartuzumab & [88] \\
\hline \multirow[t]{9}{*}{$\begin{array}{l}\text { Hepatocellular } \\
\text { carcinoma }\end{array}$} & $\begin{array}{l}\text { VEGFR, BRAF, } \\
\text { KIT, RET, PDGFR }\end{array}$ & $\begin{array}{l}\text { Sunitinib } \\
\text { Linifanib }\end{array}$ & {$[55,56]$} \\
\hline & FGFR & Brivanib & {$[57,58]$} \\
\hline & EGFR & Erlotinib & [59] \\
\hline & mTOR & Temsirolimus & [60] \\
\hline & \multirow[t]{3}{*}{ c-MET } & Foretinib & [82] \\
\hline & & Tivantinib & [83] \\
\hline & & Cabozantinib & [84] \\
\hline & MEK & Selumetinib & {$[89,90]$} \\
\hline & IGF/IGFR & Cixutumumab & [91] \\
\hline
\end{tabular}

pathways and different expressions of EGF receptors. Lapatinib is another TKI, which has been evaluated in Phase II studies for the treatment of aGC. However, it achieved modest clinical benefit $[64,65]$. In the Phase III LOGIC study, the combination therapy of lapatinib with capecitabine and oxaliplatin in comparison with chemotherapy alone as first-line treatment for HER2-positive patients didn't meet the primary end point (mOS 12.2 vs 10.5 months; HR: 0.91; 95\% CI: 0.73-1.12; $\mathrm{p}=0.35)$. Pre-specified subgroup analyses showed significant improvements in OS in Asian patients (HR: 0.68) and those under 60 years (HR: 0.69) [66]. Data of the TYTAN trial have been recently presented at the 2013 American Society of Clinical Oncology GI symposium. In this Asiatic study, lapatinib has been evaluated in combination with paclitaxel versus paclitaxel alone in pretreated HER2 amplified aGCs. OS improvement was not statistically significant in the intention-to-treat population (11.0 vs 8.9 months; HR: 0.84; $\mathrm{p}=0.2088$ ) 
while in the Chinese subgroup of HER2-positive patients, this improvement was higher [67].

In many Phase II trials, the association of cetuximab with chemotherapy has been shown to have some efficacy [68,69] and based on the increase of RR in previous studies, Richards et al. evaluated in a Phase II trial the combination of cetuximab and chemotherapy with docetaxel plus oxaliplatin (DOCOX) versus chemotherapy alone in patients affected with metastatic gastric and GEJ adenocarcinoma. Primary end point (PFS) was not met and the addition of cetuximab didn't improve either PFS or OS. They obtained a minimal improvement in ORR in the combination arm (38 vs $26 \%$; 95\% CI) without a real clinical benefit and with increased toxicity, dose reductions and delays. Although the incidence of KRAS mutations in gastric cancer is really low, patients in this trial were unselected for RAS status and this may be a reason for the lack of efficacy and the high toxicity rates [70]. According to the poor results of the previous studies, data from the EXPAND trial, a Phase III randomized study, confirmed that to date the association of cetuximab with chemotherapy for the treatment of aGC has not provided clinical benefits and that standard chemotherapy is still the backbone [71]. In the REAL3 trial, 553 patients with advanced esophagogastric cancer were randomized to receive chemotherapy (epirubicin, oxaliplatin and capecitabine) or reduced-dose chemotherapy plus panitumumab. The addition of panitumumab provided a reduction of mOS from 11.3 months (95\% CI: 9.6-13.0) with standard chemotherapy alone, to 8.8 months for the combination $(95 \%$ CI: 7.7-9.8) [72].

Data from various Phase II studies suggested that the use of bevacizumab in association with chemotherapy is a promising combination for patients with advanced GC and GEJ tumors with a range of objective response of $65-67 \%$ and survival of 12.3-16.8 months, laying the basis for further larger trials $[73,74]$. In fact, in the AVAGAST study, a multicentric randomized placebo controlled Phase III trial, 773 patients with advanced gastric or GEJ cancer have been treated with cisplatin and capecitabine and randomized to receive in addition bevacizumab or placebo. The mOS was 12.1 months in the bevacizumab arm versus 10.1 for placebo, but this difference was not significant (HR: 0.87; $\mathrm{p}=0.1002)$ and the trial didn't meet the primary end point. Nonetheless, both median PFS (6.7 vs 5.3 months; HR: 0.80 ; p. 0037) and ORR (46.0 vs $37.4 \%$; p. 0315) were significantly improved with bevacizumab versus placebo [75]. In a post hoc subgroup analysis, Shah et al. have also shown that the addition of bevacizumab to chemotherapy in European and American patients had significantly improved OS in patients with diffuse disease (mOS 6.5 vs 9.9 months; HR: 0.68 ) and in a marginal way in those with distal disease (mOS 9.0 vs 11.7 months; HR: 0.72). Combining the data of the two subtypes of cancer, there was a significant reduction of the risk of death, while Asiatic patients with any type of disease showed no significant benefit from the combination of bevacizumab to chemotherapy [76]. In another analysis of the AVAGAST trial by Van Cutsem et al., it has been demonstrated that patients with high plasma VEGF-A levels show a trend toward improved OS versus patients with low VEGF-A levels. Patients with low baseline expression of neuropilin-1 also showed a trend toward improved OS versus patients with high neuropilin-1 expression. For both biomarkers, subgroup analyses demonstrated significance only in patients from non-Asian regions [77].

Ramucirumab is a fully human $\mathrm{mAb}$ directed against the extracellular VEGF-binding domain of VEGFR-2. In the REGARD study, a Phase III, randomized double-blind trial, ramucirumab monotherapy plus best supportive care (BSC) has been compared with BSC plus placebo as second-line therapy for patients with aGC. The mOS was higher for patients in the experimental arm (5.2 vs 3.8 months; HR: $0.776 ; \mathrm{p}=0.0473)$ such as PFS (2.1 vs 1.3 months; HR: $0.483 ; \mathrm{p}<0.0001)$ and the most frequent grade $\geq 3$ adverse effects were hypertension, anemia, abdominal pain, ascites and fatigue. Efficacy and safety of ramucirumab shown in this trial seem to be promising, although second-line chemotherapy showed a similar survival benefit over BSC [78]. Results of the RAINBOW trial, a randomized multicenter double-blind, placebo-controlled trial, have been presented at the 2014 American Society of Clinical Oncology GI symposium. Ramucirumab has been evaluated in combination with paclitaxel compared with paxlitaxel plus BSC in 665 patients with metastatic GEJ and gastric adenocarcinoma in secondline treatment after a first-line therapy with platinum- and fluoropyrimidine-containing combination therapy. The trial met the primary end point obtaining an improvement in mOS for the ramucirumab arm (9.63 vs 7.36 months; HR: $0.87 ; 95 \%$ CI: $0.678-0.962 ; \mathrm{p}=0.0169)$ and in PFS (4.40 vs 2.86 months; HR: 0.635 ; 95\% CI: $0.536-0.752$; $\mathrm{p}<0.0001$ ) with a higher ORR (28 vs $16 \%$; $\mathrm{p}=0.0001$ ) and an acceptable toxicity profile [79].

\section{The finding of further pathways to target}

The c-MET proto-oncogene encodes for a surface receptor whose natural activating ligand is HGF [80]. Its activation is involved in cell diffusion, invasion and provides protection from apoptosis and stimulates angiogenesis [81]. First promising data of activity of foretinib, a multi-target c-MET TKI, came from a Phase I/II trial in which it produced an ORR of $24 \%$, with a median TTP of 4.2 months and mOS of 15.7 months for patients affected by advanced HCC as firstline therapy [82]. Tivantinib is a non-ATP-competitive c-MET inhibitor, which has been studied in patients with HCC after failure of sorafenib therapy in a Phase II, randomized, placebo-controlled trial whose results indicated an advantage in patients with tumors overexpressing c-MET in terms of median TTP (2.7 vs 1.4 months; HR: 0.43; $\mathrm{p}=0.03)$ and $\operatorname{mOS}(7.2$ vs 3.8 months; HR: 0.38; $\mathrm{p}=0.01)$ [83]. This new c-MET TKI is currently being evaluated in a Phase III, placebo-controlled, randomized study for 
patients with c-MET overexpressing HCCs after failure of sorafenib; the study is still recruiting.

Another molecule under study is cabozantinib, a nonselective TKI directed versus c-MET, VEGFR-2 and RET. Cabozantinib showed promising results in patients with HCC, despite the small number of patients enrolled in the Phase II study conduced by Verslype et al. [84] c-MET overexpression occurs also in almost $21 \%$ of gastric cancers and it seems to represent a negative prognostic factor [85]. A Phase II trial evaluated the use of foretinib as monotherapy in patients with metastatic gastric cancer with poor responses [86]. This study shows that single-agent foretinib lacks efficacy in unselected patients with aGC and selection according to MET amplification would be needed.

Rilotumumab is a fully humanized anti-HGF mAb, which has been studied in a Phase II trial in combination with epirubicin-cisplatin-capecitabine chemotherapy (ECX) for patients with aGC. It showed a trend toward improved survival in patients with MET overexpression and treated with the addition of rilotumumab to chemotherapy, whereas the addition was unfavorable in those with low MET expression [87]. These preliminary data gave the start to the Phase III trial RILOMET-1, which is still ongoing.

Onartuzumab is another anti-Met $\mathrm{mAb}$ and it is being studied in a Phase III trial [88] that will evaluate the efficacy and safety of the drug in combination with mFOLFOX6 in patients with metastatic HER2-negative and MET-positive adenocarcinoma of the stomach or GEJ. MEK represents another molecular target, which has been evaluated in various solid tumors such as malignant melanoma and non-small-cell lung cancer.

Selumetinib, a MEK inhibitor, in monotherapy or in combination with sorafenib for HCC patients, seems to have some activity, but findings are still preliminary and need to be confirmed [89,90]. IGF and IGF-1R-targeted molecules have been also evaluated in monotherapy or in combination with sorafenib for patients with HCC, although their unfavorable toxicity profiles is limiting the clinical development [91,92].

\section{Expert commentary}

Fifteen years ago, most metastatic GI tumors were difficult to treat with systemic therapy and in general prognosis was poor. Over the years, improvement was achieved with the introduction of newer cytotoxic agents such as irinotecan, oxaliplatin and taxanes. Especially in mCRC, the gain in OS has been substantial. The mOS increased from 6 to more than 20 months.

With better knowledge of tumor biology and the interaction between tumor and environment, a new class called targeted drugs was developed. The original idea for their use was that focusing on a specific target would translate into more efficacy and less toxicity (meaning a better therapeutic index). The first targeted drug introduced in GI tumors was an unexpected success. Imatinib in metastatic GI stromal tumors completely changed the management of these tumors. This was the first systemic therapy, which showed a sizeable anti-tumor activity.

In other GI tumors, interest was mainly focused on the 'growth' and 'vascular' pathways. Ten years ago, we had the introduction of bevacizumab in the treatment of mCRC. Afterward, relevant anti-tumor activity was shown with the use of cetuximab and panitumumab, both EGFR inhibitors. Although the progress per new drug was small, the overall benefit in OS was important. Besides the introduction of these new drugs, we experienced the role of biomarkers in GI tumors. First KRAS and more recently RAS testing influence dramatically our treatment algorithm in mCRC. Later, both small molecules and antibodies showed activity in other GI tumors such as gastric, neuroendocrine tumors and HCC.

What brings the future?

- in most of the GI tumors still a medical need exists for new drugs;

- the determination of the best positioning of targeted therapy in the treatment algorithm;

- understanding the tumor biology must form the base for combination treatment with targeted therapy;

- further personalization of treatment is necessary, based both on tumor and patient characteristics.

\section{Five-year view}

In our opinion, the next 5 years will see the development of many new therapeutic strategies in the landscape of medical treatment of solid tumors, including those of the GI tract. In particular, we are waiting for more concrete data about the activity of the new molecules with tyrosine kinase inhibition activity. Many of the new knowledge on biology of GI tumors derives from retrospective analyses of studies conducted in patients, which often were not rigorously selected. New prospective studies are needed in this field to identify new biomarkers and to better explain the role and prognostic significance of those that we have discovered recently.

The identification of new targets will provide soon new therapeutic strategies. Namely, c-MET, RET, MEK and IGF/ IGFR will represent both further molecules to target and predictive factors to test to tailor cancer treatment.

\section{Financial \& competing interests disclosure}

The authors have no relevant affiliations or financial involvement with any organization or entity with a financial interest in or financial conflict with the subject matter or materials discussed in the manuscript. This includes employment, consultancies, honoraria, stock ownership or options, expert testimony, grants or patents received or pending or royalties.

No writing assistance was utilized in the production of this manuscript. 


\section{Key issues}

- The introduction of targeted therapies radically changed the approach of oncologists in the treatment of the cancers of the gastrointestinal tract.

- The addition of anti-VEGF, bevacizumab and anti-EGFR, cetuximab/panitumumab, to chemotherapy, significantly improved the survival rates of metastatic colorectal cancer patients.

- Both small molecules and antibodies showed activity in other gastrointestinal (Gl) tumors, such as gastric, neuroendocrine tumors and hepatocellular carcinoma and are already available for clinical use in everyday practice.

- We now have molecular markers, whose identification has become mandatory for proper treatment planning in oncology.

- First KRAS and more recently RAS testing influence dramatically our treatment algorithm in metastatic colorectal cancer.

- A great number of new targeted agents are actually under preclinical and clinical development (i.e., MET inhibitors, MEK inhibitors, etc.). Furthermore, an antibody-drug conjugate, such as trastuzumab emtansine, is under preclinical investigation in HER2-positive gastric cancer cell lines in vitro and in vivo.

- Some of these new drugs, which are under development, were studied to improve the effects of previous agents on targets whose impairments have been already known to be related to cancer development and progression.

- Researchers worldwide are looking for further molecular pathways involved in Gl tumors and a great number of new targeted agents are actually under investigation.

- Nowadays, targeted therapy is part of daily life of patients and physicians who treat those patients with GI tumors, but much more progress can be made.

\section{References}

1. Kim KJ, Li B, Winer J, et al. Inhibition of vascular endothelial growth factor-induced angiogenesis suppresses tumour growth in vivo. Nature 1993;362:841-4

2. Shalaby F, Rossant J, Yamaguchi TP, et al. Failure of blood-island formation and vasculogenesis in Flk-1-deficient mice. Nature 1995;376:62-6

3. Kawasaki T, Kitsukawa T, Bekku Y, et al. A requirement for neuropilin-1 in embryonic vessel formation. Development 1999; 126:4895-902

4. Fong GH, Rossant J, Gertsenstein M, Breitman ML. Role of the Flt-1 receptor tyrosine kinase in regulating the assembly of vascular endothelium. Nature 1995;376: 66-70

5. Marti HH, Risau W. Angiogenesis in ischemic disease. Thromb Haemost 1999;82 (Suppl 1):44-52

6. Robinson CJ, Stringer SE. The splice variants of vascular endothelial growth factor (VEGF) and their receptors. J Cell Sci 2001;114:853-65

7. Ryuto M, Ono M, Izumi $\mathrm{H}$, et al. Induction of vascular endothelial growth factor by tumor necrosis factor alpha in human glioma cells. Possible roles of SP-1. J Biol Chem 1996;271:28220-8

8. Stavri GT, Zachary IC, Baskerville PA, et al. Basic fibroblast growth factor upregulates the expression of vascular endothelial growth factor in vascular smooth muscle cells. Synergistic interaction with hypoxia. Circulation 1995;92:11-14

9. Shweiki D, Neeman M, Itin A, Keshet E. Induction of vascular endothelial growth factor expression by hypoxia and by glucose deficiency in multicell spheroids: implications for tumor angiogenesis. Proc Natl Acad Sci USA 1995;92:768-72

10. Forsythe JA, Jiang $\mathrm{BH}$, Iyer $\mathrm{NV}$, et al. Activation of vascular endothelial growth factor gene transcription by hypoxia-inducible factor 1 . Mol Cell Biol 1996;16:4604-13

11. Ema M, Taya S, Yokotani N, et al. A novel bHLH-PAS factor with close sequence similarity to hypoxia-inducible factor 1alpha regulates the VEGF expression and is potentially involved in lung and vascular development. Proc Natl Acad Sci USA 1997;94:4273-8

12. Ikeda E, Achen MG, Breier G, Risau W. Hypoxia-induced transcriptional activation and increased mRNA stability of vascular endothelial growth factor in C6 glioma cells. J Biol Chem 1995;270:19761-6

13. Stein I, Itin A, Einat $P$, et al. Translation of vascular endothelial growth factor mRNA by internal ribosome entry: implications for translation under hypoxia. Mol Cell Biol 1998;18:3112-19

14. Gerber HP, McMurtrey A, Kowalski J, et al. Vascular endothelial growth factor regulates endothelial cell survival through the phosphatidylinositol 3'-kinase/Akt signal transduction pathway. Requirement for Flk-
1/KDR activation. J Biol Chem 1998;273: 30336-43

15. Bronte G, Terrasi M, Rizzo S, et al. EGFR genomic alterations in cancer: prognostic and predictive values. Front Biosci (Elite Ed) 2011;3:879-87

16. Russo A, Rizzo S, Bronte G, et al. The long and winding road to useful predictive factors for anti-EGFR therapy in metastatic colorectal carcinoma: the KRAS/BRAF pathway. Oncology 2009;77(Suppl 1):57-68

17. Poole EM, Curtin K, Hsu L, et al. Genetic variability in EGFR, Src and HER2 and risk of colorectal adenoma and cancer. Int J Mol Epidemiol Genet 2011;2:300-15

18. Engelman JA, Luo J, Cantley LC. The evolution of phosphatidylinositol 3-kinases as regulators of growth and metabolism. Nat Rev Genet 2006;7:606-19

19. Silvestris N, Tommasi S, Petriella D, et al. The dark side of the moon: the PI3K/ PTEN/AKT pathway in colorectal carcinoma. Oncology 2009;77(Suppl 1): 69-74

20. Kabbinavar FF, Schulz J, McCleod M, et al. Addition of bevacizumab to bolus fluorouracil and leucovorin in first-line metastatic colorectal cancer: results of a randomized phase II trial. J Clin Oncol 2005;23:3697-705

21. Hurwitz H, Fehrenbacher L, Novotny W, et al. Bevacizumab plus irinotecan, fluorouracil, and leucovorin for metastatic colorectal cancer. N Engl J Med 2004;350: 2335-42 
22. Rizzo S, Bronte G, Fanale D, et al. Prognostic vs predictive molecular biomarkers in colorectal cancer: is KRAS and BRAF wild type status required for anti-EGFR therapy? Cancer Treat Rev 2010;36(Suppl 3):S56-61

23. Van Cutsem E, Köhne CH, Láng I, et al. Cetuximab plus irinotecan, fluorouracil, and leucovorin as first-line treatment for metastatic colorectal cancer: updated analysis of overall survival according to tumor KRAS and BRAF mutation status. J Clin Oncol 2011;29:2011-19

24. Douillard JY, Oliner KS, Siena S, et al. Panitumumab-FOLFOX4 treatment and RAS mutations in colorectal cancer. N Engl J Med 2013;369:1023-34

25. Bokemeyer C, Bondarenko I, Makhson A, et al. Fluorouracil, leucovorin, and oxaliplatin with and without cetuximab in the first-line treatment of metastatic colorectal cancer. J Clin Oncol 2009;27: 663-71

26. Tveit KM, Guren T, Glimelius B, et al. Phase III trial of cetuximab with continuous or intermittent fluorouracil, leucovorin, and oxaliplatin (Nordic FLOX) versus FLOX alone in first-line treatment of metastatic colorectal cancer: the NORDIC-VII study. J Clin Oncol 2012;30:1755-62

27. Maughan TS, Adams RA, Smith CG, et al. Addition of cetuximab to oxaliplatin-based first-line combination chemotherapy for treatment of advanced colorectal cancer: results of the randomised phase $3 \mathrm{MRC}$ COIN trial. Lancet 2011;377:2103-14

28. Rolfo C, Russo A, Santini D, et al. Dilemma in metastatic colorectal cancer: VEGF versus EGRF targeting. Expert Opin Ther Targets 2013;17:869-71

29. Bennouna J, Sastre J, Arnold D, et al. Continuation of bevacizumab after first progression in metastatic colorectal cancer (ML18147): a randomised phase 3 trial. Lancet Oncol 2013;14:29-37

30. Santini D, Vincenzi B, Addeo R, et al. Cetuximab rechallenge in metastatic colorectal cancer patients: how to come away from acquired resistance? Ann Oncol 2012;23:2313-18

31. Giantonio BJ, Catalano PJ, Meropol NJ, et al. Bevacizumab in combination with oxaliplatin, fluorouracil, and leucovorin (FOLFOX4) for previously treated metastatic colorectal cancer: results from the Eastern Cooperative Oncology Group Study E3200. J Clin Oncol 2007;25:1539-44

32. Van Cutsem E, Tabernero J, Lakomy R, et al. Addition of aflibercept to fluorouracil, leucovorin, and irinotecan improves survival in a phase III randomized trial in patients with metastatic colorectal cancer previously treated with an oxaliplatin-based regimen. J Clin Oncol 2012;30:3499-506

33. Peeters M, Price TJ, Cervantes A, et al. Final results from a randomized phase 3 study of FOLFIRI $\{+/-\}$ panitumumab for second-line treatment of metastatic colorectal cancer. Ann Oncol 2014;25:107-16

34. Grothey A, Van Cutsem E, Sobrero A, et al. Regorafenib monotherapy for previously treated metastatic colorectal cancer (CORRECT): an international, multicentre, randomised, placebo-controlled, phase 3 trial. Lancet 2013;381:303-12

35. Heinemann V, Von Weikersthal LF, et al. DTe. Randomized comparison of FOLFIRI plus cetuximab versus FOLFIRI plus bevacizumab as first-line treatment of KRAS wild-type metastatic colorectal cancer: German AIO study KRK-0306 (FIRE-3). ASCO Annual Meeting; 2013; Chicago. J Clin Oncol 2013

36. Wagner AD, Grothe W, Haerting J, et al. Chemotherapy in advanced gastric cancer: a systematic review and meta-analysis based on aggregate data. J Clin Oncol 2006;24: 2903-9

37. Wang S, Zheng G, Chen L, Xiong B. Effect of HER-2/neu over-expression on prognosis in gastric cancer: a meta-analysis. Asian Pac J Cancer Prev 2011;12:1417-23

38. Bang YJ, Van Cutsem E, Feyereislova A, et al. Trastuzumab in combination with chemotherapy versus chemotherapy alone for treatment of HER2-positive advanced gastric or gastro-oesophageal junction cancer (ToGA): a phase 3, open-label, randomised controlled trial. Lancet 2010;376:687-97

39. Llovet JM, Ricci S, Mazzaferro V, et al. Sorafenib in advanced hepatocellular carcinoma. N Engl J Med 2008;359: 378-90

40. Cheng AL, Kang YK, Chen Z, et al. Efficacy and safety of sorafenib in patients in the Asia-Pacific region with advanced hepatocellular carcinoma: a phase III randomised, double-blind, placebo-controlled trial. Lancet Oncol 2009; 10:25-34

41. Chiu J, Tang YF, Yao TJ, et al. The use of single-agent sorafenib in the treatment of advanced hepatocellular carcinoma patients with underlying Child-Pugh B liver cirrhosis: a retrospective analysis of efficacy, safety, and survival benefits. Cancer 2012;118:5293-301
42. Knosel T, Chen Y, Altendorf-Hofmann A, et al. High KIT and PDGFRA are associated with shorter patients survival in gastroenteropancreatic neuroendocrine tumors, but mutations are a rare event. J Cancer Res Clin Oncol 2012;138:397-403

43. Murakumo Y, Jijiwa M, Asai N, et al. RET and neuroendocrine tumors. Pituitary 2006;9:179-92

44. Demirkan BH, Eriksson B. Systemic treatment of neuroendocrine tumors with hepatic metastases. Turk J Gastroenterol 2012;23:427-37

45. Raymond E, Dahan L, Raoul JL, et al. Sunitinib malate for the treatment of pancreatic neuroendocrine tumors. $\mathrm{N}$ Engl J Med 2011;364:501-13

46. Yao JC, Shah MH, Ito T, et al. Everolimus for advanced pancreatic neuroendocrine tumors. N Engl J Med 2011;364:514-23

47. Pommier RF, Wolin EM, Panneerselvam A. Impact of prior chemotherapy on progression-free survival in patients (pts) with advanced pancreatic neuroendocrine tumors (pNET): Results from the RADIANT-3 trial. ASCO Annual Meeting Abstracts, J Clin Oncol 2011. 29(15 Suppl); 4103

48. Shah MH, Lombard-Bohas C, Ito T, et al. Everolimus in patients with advanced pancreatic neuroendocrine tumors (pNET): Impact of somatostatin analog use on progression-free survival in the RADIANT-3 trial. 2011 ASCO Annual Meeting Abstracts, J Clin Oncol 2011. 29(15 Suppl); 4010

49. Altomare I, Bendell JC, Bullock KE, et al. A phase II trial of bevacizumab plus everolimus for patients with refractory metastatic colorectal cancer. Oncologist 2011;16:1131-7

50. Matar P, Rojo F, Cassia R, et al. Combined epidermal growth factor receptor targeting with the tyrosine kinase inhibitor gefitinib (ZD1839) and the monoclonal antibody cetuximab (IMC-C225): superiority over single-agent receptor targeting. Clin Cancer Res 2004;10:6487-501

51. Weickhardt AJ, Price TJ, Chong G, et al Dual targeting of the epidermal growth factor receptor using the combination of cetuximab and erlotinib: preclinical evaluation and results of the phase II DUX study in chemotherapy-refractory, advanced colorectal cancer. J Clin Oncol 2012;30: 1505-12

52. Carrato A, Swieboda-Sadlej A, Staszewska-Skurczynska M, et al. Fluorouracil, leucovorin, and irinotecan plus 
either sunitinib or placebo in metastatic colorectal cancer: a randomized, phase III trial. J Clin Oncol 2013;31:1341-7

53. Tabernero J, Garcia-Carbonero R, Cassidy J, et al. Sorafenib in combination with oxaliplatin, leucovorin, and fluorouracil (modified FOLFOX6) as first-line treatment of metastatic colorectal cancer: the RESPECT trial. Clin Cancer Res 2013;19: 2541-50

54. Wolpin BM, Ng K, Zhu AX, et al. Multicenter phase II study of tivozanib (AV-951) and everolimus (RAD001) for patients with refractory, metastatic colorectal cancer. Oncologist 2013;18:377-8

55. Cheng AL, Kang Y, Lin D, et al. Phase III trial of sunitinib $(\mathrm{Su})$ versus sorafenib (So) in advanced hepatocellular carcinoma (HCC). J Clin Oncol 2011; 29(Suppl); abstr 4000

56. Cainap C, Qin S, Huang WT, et al. Phase III trial of linifanib versus sorafenib in patients with advanced hepatocellular carcinoma (HCC). J Clin Oncol 2013; 30(Suppl 34); abstr 249

57. Johnson P, Qin S, Park JW, et al. Brivanib (BRI) versus sorafenib (SOR) as first-line therapy in patients with unresectable, advanced hepatocellular carcinoma (HCC): results from the phase 3 BRISK-FL study. In 63rd Annual Meeting of the American Association for the Study of Liver Disease 2012; abstr LB-6

58. Llovet JM, Decaens T, Raoul JL, et al. Brivanib versus placebo in patients with advanced hepatocellular carcinoma (HCC) who failed or were intolerant to sorafenib: results from the phase 3 BRISK-PS study. In 47th International Liver Congress (EASL) 2012; abstr 1398

59. Zhu AX, Rosmorduc O, Evans J, et al. SEARCH: a phase III, randomized, double-blind, placebo-controlled trial of sorafenib plus erlotinib in patients with hepatocellular carcinoma (HCC). In ESMO Congress 2012; abstr 917

60. Chelis L, Deftereos S, Xenidis N, et al. Bevacizumab plus temsirolimus as second-line treatment for advanced hepatocellular carcinoma (HCC). J Clin Oncol 2012;30(Suppl); abstr e14567

61. Dragovich T, McCoy $S$, Fenoglio-Preiser CM, et al. Phase II trial of erlotinib in gastroesophageal junction and gastric adenocarcinomas: SWOG 0127. J Clin Oncol 2006;24:4922-7

62. Wainberg ZA, Lin LS, DiCarlo B, et al. Phase II trial of modified FOLFOX6 and erlotinib in patients with metastatic or advanced adenocarcinoma of the oesophagus and gastro-oesophageal junction. $\mathrm{Br}$ J Cancer 2011;105:760-5

63. Rodriguez CP, Adelstein DJ, Rice TW, et al. A phase II study of perioperative concurrent chemotherapy, gefitinib, and hyperfractionated radiation followed by maintenance gefitinib in locoregionally advanced esophagus and gastroesophageal junction cancer. J Thorac Oncol 2010;5: 229-35

64. Iqbal S, Goldman B, Lenz HJ, et al. S0413: A phase II SWOG study of GW572016 (lapatinib) as first line therapy in patients (pts) with advanced or metastatic gastric cancer. J Clin Oncol 2007;25; Abstract 4621

65. Hecht JR, Urba SG, Koehler M, et al. Lapatinib monotherapy in recurrent upper gastrointestinal malignancy: Phase II efficacy and biomarker analyses. In Gastrointestinal Cancers Symposium (ASCO) 2008; Abstract 43

66. Hecht J, Bang YJ, Qin S, et al. Lapatinib in combination with capecitabine plus oxaliplatin (CapeOx) inHER2-positive advanced or metastatic gastric, esophageal, or gastro-esophageal adenocarcinoma (AC): the TRIO-013/LOGiC Trial. J Clin Oncol 2013;31(Suppl 1); abstr LBA4001

67. Bang Y-J. The Chinese subgroup from a randomized phase III study of lapatinib in combination with weekly paclitaxel versus weekly paclitaxel alone as second-line treatment of HER2-amplified advanced gastric cancer (AGC) in Asian countries. J Clin Oncol 2012;30(suppl 34; abstr 11

68. Moehler M, Mueller A, Trarbach T, et al. Cetuximab with irinotecan, folinic acid and 5-fluorouracil as first-line treatment in advanced gastroesophageal cancer: a prospective multi-center biomarkeroriented phase II study. Ann Oncol 2011;22:1358-66

69. Kim C, Lee JL, Ryu MH, et al. A prospective phase II study of cetuximab in combination with XELOX (capecitabine and oxaliplatin) in patients with metastatic and/or recurrent advanced gastric cancer. Invest New Drugs 2011;29:366-73

70. Richards D, Kocs DM, Spira AI, et al. Results of docetaxel plus oxaliplatin $(\mathrm{DOCOX}) \pm$ cetuximab in patients with metastatic gastric and/or gastroesophageal junction adenocarcinoma: results of a randomised Phase 2 study. Eur J Cancer 2013;49:2823-31

71. Lordick F, Kang YK, Chung HC, et al. Capecitabine and cisplatin with or without cetuximab for patients with previously untreated advanced gastric cancer (EXPAND): a randomised, open-label phase 3 trial. Lancet Oncol 2013;14: 490-9

72. Waddell T, Chau I, Cunningham D, et al Epirubicin, oxaliplatin, and capecitabine with or without panitumumab for patients with previously untreated advanced oesophagogastric cancer (REAL3): a randomised, open-label phase 3 trial. Lancet Oncol 2013;14:481-9

73. Shah MA, Ramanathan RK, Ilson DH, et al. Multicenter phase II study of irinotecan, cisplatin, and bevacizumab in patients with metastatic gastric or gastroesophageal junction adenocarcinoma. J Clin Oncol 2006;24:5201-6

74. Shah MA, Jhawer M, Ilson DH, et al. Phase II study of modified docetaxel, cisplatin, and fluorouracil with bevacizumab in patients with metastatic gastroesophageal adenocarcinoma. J Clin Oncol 2011;29: 868-74

75. Ohtsu A, Shah MA, Van Cutsem E, et al. Bevacizumab in combination with chemotherapy as first-line therapy in advanced gastric cancer: a randomized, double-blind, placebo-controlled phase III study. J Clin Oncol 2011;29:3968-76

76. Shah MA, Van Cutsem E, Kang YK, et al. Survival analysis according todisease subtype in AVAGAST: first-line capecitabine and cisplatin plus bevacizumab (BEV) or placebo in patients with advanced gastric cancer. ASCO Meet Abstr 2012; 30(Suppl 4):5

77. Van Cutsem E, de Haas S, Kang YK, et al. Bevacizumab in combination with chemotherapy as first-line therapy in advanced gastric cancer: a biomarker evaluation from the AVAGAST randomized phase III trial. J Clin Oncol 2012;30: 2119-27

78. Fuchs CS, Tomasek J, Yong CJ, et al. Ramucirumab monotherapy for previously treated advanced gastric or gastro-oesophageal junction adenocarcinoma (REGARD): an international, randomised, multicentre, placebo-controlled, phase 3 trial. Lancet 2014;383:31-9

79. Wilke H, Van Cutsem E, Oh SC, et al. RAINBOW: A global, phase III, randomized, double-blind study of ramucirumab plus paclitaxel versus placebo plus paclitaxel in the treatment of metastatic gastroesophageal junction (GEJ) and gastric adenocarcinoma following disease progression on first-line platinum- and fluoropyrimidine-containing combination therapy rainbow IMCL CP12-0922 
(I4T-IE-JVBE). In 2014 Gastrointestinal Cancers Symposium; Abstract No: LBA7

80. Gherardi E, Birchmeier W, Birchmeier C, Vande Woude G. Targeting MET in cancer: rationale and progress. Nat Rev Cancer 2012;12:89-103

81. Comoglio PM, Giordano S, Trusolino L. Drug development of MET inhibitors: targeting oncogene addiction and expedience. Nat Rev Drug Discov 2008;7: 504-16

82. Yau TC, Sukeepaisamjaroen W, Chao Y, et al. A phase I/II study of foretinib, an oral multikinase inhibitor targeting MET, RON, AXL, TIE-2, and VEGFR in advanced hepatocellular carcinoma (HCC). J Clin Oncol 2012;30(Suppl); abstr 4108

83. Santoro A, Rimassa L, Borbath I, et al. Tivantinib for second-line treatment of advanced hepatocellular carcinoma: a randomised, placebo-controlled phase 2 study. Lancet Oncol 2013;14:55-63

84. Verslype C, Cohn AL, Kelley RK, et al. Activity of cabozantinib (XL184) in hepatocellular carcinoma: Results from a phase II randomized discontinuation trial
(RDT). J Clin Oncol 2012; 30(Suppl); abstr 4007

85. Lee J, Seo JW, Jun HJ, et al. Impact of MET amplification on gastric cancer: possible roles as a novel prognostic marker and a potential therapeutic target. Oncol Rep 2011;25:1517-24

86. Shah MA, Wainberg ZA, Catenacci DV, et al. Phase II study evaluating 2 dosing schedules of oral foretinib (GSK1363089), cMET/VEGFR2 inhibitor, in patients with metastatic gastric cancer. PLoS One 2013;8: e54014

87. Oliner KS, Tang R, Anderson A, et al. Evaluation of MET pathway biomarkers in a phase II study of rilotumumab (R AMG $102)$ or placebo(P) in combination with epirubicin, cisplatin, and capecitabine (ECX) inpatients (pts) with locally advanced or metastatic gastric $(\mathrm{G})$ or esophagogastric junction (EGJ) cancer. ASCO Meeting 2012;30(Suppl 15); Abstr 4005

88. ClinicalTrials.gov Identifier. NCT01662869. Available from: http:// clinicaltrials.gov/show/NCT01662869
89. O'Neil BH, Goff LW, Kauh JS, et al. Phase II study of the mitogen-activated protein kinase $1 / 2$ inhibitor selumetinib in patients with advanced hepatocellular carcinoma. J Clin Oncol 2011;29:2350-6

90. Choo SP, Ng QS, Chen WJJ, et al. A phase I/II study of AZD6244 in combination with sorafenib in advanced hepatocellular carcinoma. J Clin Oncol 2012; 30(Suppl); abstr 4100

91. Abou-Alfa GK, Gansukh B, Chou JF, et al. Phase II study of cixutumumab (IMC-A12, NSC742460; C) in hepatocellular carcinoma (HCC). J Clin Oncol 2011; 29(Suppl 4); abstr 4043

92. Barok M, Tanner M, Koninki K, Isola J. Trastuzumab-DM1 is highly effective in preclinical models of HER2-positive gastric cancer. Cancer Lett 2011;306:171-9

93. ClinicalTrials.gov Identifier. NCT01697072. Available from: http:// clinicaltrials.gov/show/NCT01697072 